\title{
Pratiques
}

Linguistique, littérature, didactique

\section{La cohérence textuelle : pratiques des enseignants et théories de référence}

\section{Fabienne Rondelli}

\section{(2) OpenEdition}

\section{Journals}

\section{Édition électronique}

URL : http://journals.openedition.org/pratiques/1505

DOI : 10.4000/pratiques.1505

ISSN : 2425-2042

\section{Éditeur}

Centre de recherche sur les médiations (CREM)

\section{Édition imprimée}

Date de publication : 15 juin 2010

Pagination : 55-84

\section{Référence électronique}

Fabienne Rondelli, «La cohérence textuelle : pratiques des enseignants et théories de référence », Pratiques [En ligne], 145-146 | 2010, mis en ligne le 15 juin 2010, consulté le 30 avril 2019. URL : http:// journals.openedition.org/pratiques/1505; DOI : 10.4000/pratiques.1505 


\title{
La cohérence textuelle : pratiques des enseignants et théories de référence
}

\author{
Fabienne Rondelli
}

Université Paul Verlaine - Metz, CELTED, EA 3474

Cet article se donne pour objectif de rendre compte de quelques résultats significatifs à propos de la manière dont des professeurs des écoles jugent la cohérence de textes produits par des élèves. Nous présenterons le protocole de recherche afin que le lecteur puisse se forger une représentation assez précise des enjeux de cette étude liés 1) à la complexité de 1'objet observé (la cohérence), 2) à l'hypothèse méthodologique même qui est la nôtre. Elle se caractérise par le fait que nous ne sommes pas partie d'une théorie de référence en matière de cohérence textuelle pour l'appliquer à l'analyse d'un corpus mais que nous avons eu recours à différentes théorisations s'intéressant au texte et à la dimension transphrastique des énoncés, afin de pouvoir mieux comprendre et décrire les critères spontanés et proto-théoriques mobilisés par les professeurs des écoles.

En référence au deuxième objectif assigné à ce numéro de Pratiques, nous tenterons d'interroger les liens qui existent entre les investigations savantes, les pratiques de classe et la formation des enseignants.

Il existe, en effet, des degrés de formalisation à la base des définitions de la cohérence qui sont variables selon qu'ils relèvent :

- des définitions savantes proprement dites en matière de théories du texte selon les paradigmes théoriques auxquels ils appartiennent;

- des conceptions plus ou moins implicites, non théorisées ou incomplètement théorisées, qui fondent les jugements portés sur les textes par les enseignants.

En fonction de quoi, explorer les représentations de la notion de cohérence textuelle qui sont celles des enseignants, c'est tenter de répondre à trois questions :

- Quels sont les critères sur lesquels les professeurs des écoles s'appuient pour juger la cohérence d'un texte d'élève?

- En quoi y a-t-il accord ou écart entre les critères à usage professionnel et ceux des définitions savantes?

- En quoi la confrontation de ces deux réalités peut-elle être utile à la recherche en didactique?

Dans le cadre de cet article, nous nous attacherons à présenter nos modes d'investigation, qui nous ont permis, en tenant compte des apports théoriques en ma- 
tière de cohérence des textes, d'élaborer une grille de lecture susceptible de rendre compte des jugements des enseignants.

Nous illustrerons ensuite les conceptions implicites de la cohérence émanant des jugements portés sur des textes d'élèves à partir de quelques exemples qui nous semblent significatifs. Nous exposerons ainsi certains aspects des protothéories des enseignants.

Nous terminerons en montrant en quoi cette recherche permet de poursuivre notre réflexion sur la formation des maitres à l'évaluation de la cohérence des textes d'élèves. Nous le ferons en examinant les congruences ou les divergences qui peuvent exister entre les jugements des enseignants et les modèles descriptifs en vigueur dans les recherches sur la textualité.

\section{L'outil d'analyse des jugements de cohérence}

\subsection{Des analyses savantes non convergentes}

Pour cerner la notion de cohérence, il paraît raisonnable de partir d'une postulation très puissante : le principe de coopération de Grice (1979). Il repose sur l'idée que face à des énoncés engendrés les uns à la suite des autres, le récepteur leur attribue une logique et une cohérence justifiées par des principes de base qui président à leur production. Partant, le sujet s'efforce de construire les inférences nécessaires pour trouver le lien sémantique entre les énoncés. Cependant, deux phénomènes semblent rendre les choses un peu plus compliquées : d'une part, il arrive que le même texte soit jugé cohérent par certains destinataires et non par d'autres. Il est démontré en effet que le récepteur s'arrête plutôt à des associations de sens commun et ne fait pas spontanément d'inférences qui ne soient pas a minima prévisibles (Sperber et Wilson, 1989). D'autre part, un texte peut difficilement être traité en une seule fois, que ce soit en réception ou en production (Charolles, 1995). C'est pourquoi les scripteurs comme les lecteurs intègrent généralement des traits internes au texte lui-même qui sont constitutifs de sa cohérence et aident à la fois à la production et à la compréhension : des marques linguistiques contribuent donc à l'organisation du texte en un tout signifiant.

Au final, comment s'élabore la cohérence ? Les quelques lignes qui précèdent laissent entrevoir la difficulté d'une réponse simple et univoque ${ }^{(1)}$ du fait même de la tension permanente entre une caractérisation qui semble montrer que la cohérence est tendanciellement un fait du sujet et une position qui tend plutôt à la considérer comme tendanciellement un fait du texte.

D'un autre côté, notre objectif est de décrire et d'analyser les conceptions des enseignants, dans toutes leur variété, au travers des jugements qu'ils ont portés sur des textes d'élèves. Il nous a donc paru intéressant de concevoir un principe descriptif qui intègre les deux paramètres - du texte et du sujet - afin de pouvoir caractériser et comparer les définitions savantes et les représentations proto-théoriques des enseignants. Ce choix nous a semblé d'autant plus nécessaire que les textes évalués sont des récits, textes à la fois référentiels et fictionnels, dont l'organisation ne peut se réduire à un prototype. Cependant, choisir de mettre en perspective des points de vue complémentaires à propos de la cohérence nous a très

(1) Nous rappelons ce propos de Michel Charolles qui, il faut bien le dire, nous a perturbée au moment de l'écriture de notre thèse : Il n'est pas sûr que l'on puisse définir précisément en quoi consiste la cohérence, ni non plus qu'il faille chercher à le faire (2006:26). 
vite amenée à renoncer à toute ambition d'exhaustivité et de synthèse tant le texte est aujourd'hui l'objet d'étude de nombreux courants de recherche tels que la pragmatique du discours, l'analyse textuelle des discours, la sémantique textuelle, la psycholinguistique textuelle, l'interactionnisme socio-discursif ${ }^{(2)}$. C'est pourquoi nous avons effectué un bilan nécessairement sélectif, désireuse de mettre en valeur les conceptions divergentes de la relation texte/sujet dans la construction de la cohérence.

Sont révélatrices, à cet égard, les descriptions de la nature et des effets des liens entre des unités considérées comme les « unités textuelles de base » sur lesquelles repose le sentiment de cohérence textuelle ${ }^{(3)}$.

Trois postulats particulièrement utiles à notre projet d'analyse de jugements ont été retenus : 1) les unités de base sont discrètes, localisables et s'organisent de façon interne et hiérarchique (position de l'analyse textuelle des discours) 2) les unités de base ne sont ni discrètes ni localisables, ne s'organisent pas de façon hiérarchique mais par diffusion et sommation de formes sémantiques (position de la sémantique textuelle et interprétative) 3) les unités de base sont discrètes et s'organisent par construction-intégration sémantique (position de la psycholinguistique textuelle).

L'analyse textuelle des discours décrit les régularités qui président à l'agencement d'un texte et lui confèrent la propriété de former une unité cohésive et cohérente. Jean-Michel Adam (1999) prend appui sur le concept d' « action langagière », qui fait partie de l'appareil notionnel du psychologue interactionniste Jean-Paul Bronckart (1997), pour inviter à considérer le langage comme une production interactive associée aux activités sociales, production qui implique la constitution de représentations. Les représentations collectives progressivement constituées mais toujours en évolution permettent l'intercompréhension entre des communautés verbales. Ces pôles d'intercompréhension sont qualifiés par Bronckart de formations socio-langagières et débouchent sur les divers genres de textes (1997). Le sujet va mobiliser ses représentations en lien avec les conditions d'énonciation dans lesquelles il se trouve. Ce sera sa «base d'orientation » pour l'élaboration-compréhension d'un texte. Les textes sont donc des produits verbaux résultant de la tension entre une situation d'énonciation singulière et les régularités intertextuelles d'un genre. Ces régularités s'actualisent nécessairement dans le cadre des ressources linguistiques offertes par une langue naturelle donnée : les mondes construits par le sujet, qu'ils soient fictionnels ou ancrés dans le réel, ne sont attestables qu'à partir des formes linguistiques qui les sémiotisent, et ils sont donc solidaires de ces formes linguistiques. Il existe de ce fait une architecture interne des textes : un texte est constitué d' unités clairement identifiables qui s'articulent entre elles, qui se lient entre elles : ces unités associent des propriétés syntaxiques et sémantiques et sont le produit d'une énonciation. Ce qui signifie qu'elles s'actualisent sous la forme de trois composantes : une dimension énonciative (on parle d'un certain point de vue),

(2) «Cette capacité à générer des théories et des modèles de représentation [...] est une preuve indiscutable que le texte devient, selon le dire de F. Rastier, non plus 1'objet empirique mais 1'objet réel de la linguistique. » (Legallois 2003 : 2).

(3) Nous n'employons pas «texte» en opposition à «discours»; au contraire, nous posons, suivant les linguistes du texte, qu'un texte se définit à la fois par une dimension situationnelle, une dimension structurelle et une dimension idéationnelle. Perspective qu'André Petitjean précisait dès 1989 dans son approche de l'écrit comme objet langagier (1989:118) et qui est une base d'analyse de la plupart des chercheurs qui se sont intéressés au langage comme formation socio-discursive («les coordonnées et le statut matériel de l'énoncé font partie de ses caractères intrinsèques » par exemple selon Foucault (1969: 132)). 
une dimension référentielle (on parle de quelque chose) et une dimension argumentative (on parle avec une intention déterminée). Selon Adam (2005), chaque unité (qu'il appelle proposition-énoncé) est liée au co-texte amont et au co-texte aval par cinq types de liage : les connexions (connecteurs et organisateurs textuels), les implicitations (ellipses, présupposés, sous-entendus), les chaines d'actes de discours (les actes de discours dans un texte sont liés et hiérarchisés), les liages du signifiant (répétitions de phonèmes, syllabes, lexèmes, groupes morphosyntaxiques), les liages du signifié (anaphores, isotopies). Le texte existe si ces types de lien sont effectivement actualisés : « chacune de ces cinq opérations est un facteur de textualité, mais aucune ne suffit seule à faire d'un texte une unité cohérente ». (Adam 2005, 85). Il est cependant également nécessaire que ces opérations s'organisent en configurations identifiables : les séquences textuelles. Elles sont plus ou moins typées et ont comme caractéristiques le fait de correspondre à des catégories de relations macro-sémantiques mémorisées par imprégnation socio-culturelle. Le texte est donc un matériau verbal construit dans le cadre d'une formation langagière en référence à un genre et dont l'organisation interne, en appui sur des unités discrètes et localisables, en dénote la cohérence.

Les théories interprétatives cherchent à mettre au jour la manière dont les mots, parce qu'ils sont inscrits dans un cotexte, provoquent des effets de sens.

Lorsque François Rastier analyse la cohérence comme l' « unité d'une séquence linguistique, définie par ses relations avec son entour » (Rastier 1987, 273), il confirme l'idée qu'un texte résulte d'une tension entre des caractéristiques intrinsèques et des caractéristiques extrinsèques. Il est d'accord sur le fait que le texte est un ensemble constitué : «entre la forme matérielle du texte et ses interprétations, tout un système de normes, dont témoignent les structures textuelles, assurent la médiation indispensable » (Rastier, 2001, 23). Cependant, il développe une théorie sémantique qui s'appuie sur deux paradigmes : 1) la sémantique interprétative ne décline pas d'unités linguistiques discrètes et localisables 2) la sémantique interprétative élucide le simple par le complexe (Rastier, 2006). Ainsi est réfutée l'idée d'organisation hiérarchisée d'unités clairement identifiées qui s'articuleraient par des opérations de liage, de composition du texte à partir d'unités élémentaires qui pourraient trouver une distribution en texte. Plus exactement, il s'agit de montrer que si des unités existent et peuvent être décrites du point de vue morphosyntaxique, en aucun cas elles ne peuvent être des unités constitutives en soi du sens d'un texte.

Privés de leur contexte, si les mots et les phrases ont en général une structure morphosyntaxique identifiable, il n'en va pas de même pour leur sens, qui reste indéfiniment équivoque. Seule la connaissance du contexte proche et lointain peut guider les interprétations plausibles. (Rastier, Cavazza, Abeillé 1994 : 36).

De ce point de vue, construire un sens ne consiste pas en une opération qui s'appuierait sur le palier du mot, puis de la phrase, puis du texte, dans une sorte de construction progressive mais part du palier le plus englobant (le genre) pour attribuer du sens à la phrase et au mot.

Dans ce cadre, le rôle de l'entour d'un segment textuel est tel que l'on ne peut pas déterminer a priori quel est l'empan du texte qui va devenir une unité. Partant, ces unités sont morphosémantiques: elles n'appartiennent pas forcément à des catégories uniformes, ne sont pas forcément hiérarchisables, n'ont pas forcément de signifiant localisable comme par exemple les parties du discours. Elles s'actualisent du fait d'un parcours interprétatif et l'on ne peut analyser leur lexicalisation 
qu'après la lecture du texte. Le parcours interprétatif prend appui sur des configurations qui forment une unité de sens du fait de l'ensemble du texte.

La construction de la cohérence devient donc un parcours interprétatif, où le sens se construit dans une dynamique fondée sur une sélection thématique que réalise le récepteur à partir de scénarios intertextuels qu'il connaît. Autrement dit, chaque récepteur fait appel à sa compétence encyclopédique et intertextuelle pour interpréter un texte, en actualisant, au fur et à mesure de son avancée dans le texte, les propriétés sémantiques dont il a besoin (par exemple, dans le cadre encyclopédique du conte, l'occurrence loup activera des propriétés comme " parle », en revanche, dans un texte documentaire, le même lexème loup orientera le récepteur vers l'encyclopédie qui règle le monde « réel»). Ainsi, la place et le rôle du sujet deviennent essentiels. C'est la position d'U. Eco quand il précise que c'est le récepteur qui décide des propriétés qui doivent être activées ou narcotisées en faisant des hypothèses sur le topic ou les topics du texte (Eco, 1985). Déterminer le topic signifie avancer une hypothèse quant à une certaine régularité textuelle. Pour U. Eco, ce type de régularité est ce qui fixe « tant les limites que les conditions de cohérence d'un texte» (Eco, 1985 : 114). La cohérence interprétative consiste en une redondance assurant une homogénéité sémantique. Le texte construit donc un effet de monde. Mais si les mots ouvrent des mondes, ils le font parfois sans suivre la linéarité du texte. Ainsi, il est fréquent (particulièrement dans les textes littéraires) que le début d'un texte suggère un topic pour finalement en proposer un autre. La cohérence textuelle se construit donc progressivement, dans un double mouvement d'anticipation et de traitement rétro-actif et ne saurait se décrire en terme de lecture linéaire. On peut dire que si la représentation d'un monde est constituée par l'agencement de signifiés, elle dépasse la somme des signifiés : chaque phrase, isolée, peut s'entendre de différentes façons, mais c'est le texte dans sa globalité qui détermine la signification à privilégier dans chacune des phrases et qui institue ainsi les unités textuelles.

Les cognitivistes et psycholinguistes expliquent le processus de construction de la cohérence en montrant par quelles opérations le sujet construit du sens en opérant des liens entre le connu, le déjà lu et le nouveau « en train d'être lu ». Ils adoptent, en grande majorité, la «proposition » comme unité de base de la description des contenus sémantiques. Que veut dire «proposition» dans le cadre de la psycholinguistique? Elle s'entend comme une unité de signification : c'est donc sur le contenu du message plus que sur sa forme qu'est mis l'accent. Quel intérêt pour notre étude ? L'écriture de la suite d'un texte (tâche demandée aux élèves) implique l'organisation en mémoire du contenu sémantique du texte de départ. De même, l'évaluation d'un texte (tâche demandée aux enseignants) demande l'intégration du message écrit en un contenu de sens. Aussi, les modèles propositionnels permettent de décrire la signification des textes en s'appuyant sur un élément qui a une valeur particulière dans notre cas précis de la présence d'un texteamorce soumis aux élèves : le lexique. En effet, la signification de toute unité lexicale correspond en mémoire à une liste de propositions, c'est-à-dire à des entités référentielles (objets, êtres, idées) sur lesquelles le texte dit quelque chose (prédication). Grâce à la mémoire sémantique, les propositions sont liées entre elles sous la forme d'un réseau. La réception textuelle est forcément une activité qui se déroule dans le temps, ce qui implique que l'on s'interroge sur les moyens cognitifs à la disposition des sujets pour activer non pas une signification stable et unique, mais une signification mouvante car en cours, une signification plus ou moins provisoire. Les psycholinguistes sont d'accord sur le fait qu'en cours de lecture, des connaissances en mémoire à long terme sont activées en réseau (Per- 
ruchet, 1988, Gombert, 1997), c'est-à-dire qu'à la lecture de chaque mot, de façon largement automatisée, les sens possibles d'un mot sont activés, puis très rapidement, le sens conforme au contexte reste. Ainsi, à chaque stimulation linguistique, une signification renvoie à des significations associées. Or, cette élaboration « spontanée », automatisée est à la fois une aide et une entrave : une aide car 1'accessibilité des unités appartenant au même réseau de connaissance qu'une première unité activée se trouve accrue (soit l'activation d'un scénario, tout le réseau de connaissances à disposition du sujet serait plus facilement disponible) ; une entrave dans le sens où l'activation automatique implique la mise au premier plan d'unités non pertinentes. Intervient donc un second processus qui va exclure les éléments non pertinents. Cela veut dire que l'intégration sémantique se réalise en deux paliers : un palier horizontal et un palier vertical (Coirier et al., 1996). La composante horizontale consiste en la recherche par le sujet pour chaque information nouvelle d'une information ancienne à laquelle la rattacher. La composante verticale est plus contrôlée, car c'est celle qui conduit à l'élaboration d'une macrostructure, autrement dit, à une représentation du texte en fonction des connaissances du sujet et des scénarios mentaux à sa disposition. Ces « schémas » sont organisés en mémoire par association d'informations sur un objet, une situation, un évènement. Il est généralement précisé qu'ils ont deux fonctions essentielles : assurer la cohérence de la représentation du texte par la mise en lien des différents éléments et permettre l'anticipation et la prédiction d'éléments du texte.

Mais une autre fonction des schémas nous semble particulièrement intéressante à relever dans le cadre de notre étude : son rôle reconstructif (modèle de Spiro décrit par Coirier et al., 1996). Outre la mise en lien des informations antérieures avec les informations nouvelles, les schémas, selon Spiro (1980), peuvent être la source de reconstructions sémantiques : ainsi, s'il manque des éléments dans un texte, un sujet les intégrera parce qu'il met en lien le donné textuel avec ses propres scénarios. De même, si un texte propose des informations ne semblant pas congruentes, il est prouvé que les sujets font un rappel du texte en faisant intervenir leurs schémas et en réduisant les incohérences (Coirier et al., 1996). Le lecteur peut donc commettre des erreurs reconstructives pour tenter de réduire le désaccord entre ce qu'il lit et sa propre représentation du monde proposé par le texte.

Sur la question de la congruence, une autre théorie psychologique a retenu notre attention : la théorie de 1'intégration conceptuelle (Turner, 2000 ; Fauconnier et Turner, 2002). Il s'agit d'une théorie qui a pour ambition d'étudier scientifiquement la créativité humaine. En d'autres termes, elle tente de mettre au jour les opérations mentales qu'active un sujet pour sélectionner et intégrer de manière constructive des données qui appartiennent à des espaces, à des mondes différents pour en construire une représentation cohérente. Cette théorie montre que le sujet procède à un mélange associatif, analogique d'éléments a priori distincts pour construire une représentation cohérente d'énoncés semblant au départ sans lien. L'être humain a une capacité d'intégration conceptuelle qui lui permet de saisir le sens d'associations inédites. La théorie de l'intégration conceptuelle semble donc concerner directement la question de la construction de la cohérence textuelle. Elle peut s'actualiser à deux niveaux : 1) dans les occurrences textuelles où les énoncés apparaissent incongrus localement mais pour lesquels un lien thématique peut être facilement inféré 2) dans les occurrences textuelles où la relation de cohérence entre les énoncés paraît très difficile, voire impossible à construire. Ainsi, si la relation causale est privilégiée dans la mise en lien de deux événements, un principe complémentaire de connexion entre les idées peut être retenu pour comprendre comment le sujet interprète un texte : la relation de ressemblance $:$ le sujet a la 
capacité de mettre en lien des informations a priori incongrues ou non liées en s'appuyant sur un schéma d'interprétation préconstruit ou en inventant une relation originale.

Les trois champs théoriques convoqués montrent que la construction de la cohérence relève d'une tâche récurrente pour le lecteur : mettre en lien le « déjà lu » avec ce qui arrive. Mais ce « déjà lu » revêt deux dimensions : il est local lorsqu'il s'agit de prendre en compte le cotexte immédiat afin d'opérer un liage, il est global lorsqu'il s'agit de faire appel à l'ensemble du texte, lui-même inscrit dans un intertexte et impliquant la mémoire discursive du sujet. Ces deux dimensions impliquent d'intégrer à notre appareil méthodologique la notion de genre et très spécifiquement, la question des propriétés, qui au-delà de la singularité de chaque texte, lui confèrent un « air de famille». Pour ce faire, nous reprenons la définition de la généricité proposée par A. Petitjean (2007) :

On définira [...] la généricité comme un emboitement et une interactions de propriétés, à savoir : l'ancrage institutionnel (sphère d'activités, situation sociodiscursive de production et de réception du texte...; l'intention communicationnelle (vision, registre, disposition intentionnelle, enjeux illocutoires, buts perlocutoires.) ; le mode énonciatif (situation d'énonciation, type d'ancrage énonciatif, attitudes élocutives, formes de dialogisme...) ; le matériau de réalisation (ordre de l'oral ou de l'écrit, codage sémiotique, mise en page et mise en forme typographique pour l'écrit...) ; la macro et la micro organisation formelle (volume de la verbalisation, structure compositionnelle, agencement de séquences, procédés textuels, texture micro- linguistique...), les contenus thématiques (domaines sémantiques, macro-thèmes, réseaux lexicaux...) ; les indices para et péritextuels. (préface ou postface, titres, sous-titres...) (Petitjean 2007 : 425).

Cette dimension est particulièrement utile dans le contexte scolaire qui nous intéresse, car elle permet d'affiner notre grille d'analyse des jugements, dans le sens où si la question du lien entre la singularité du texte et son « air de famille» se pose, il est tout aussi nécessaire dans le cadre de la dialectique production- réception textuelle qui est celui de notre recherche, de permuter les termes du lien : si un texte semble appartenir à un genre, en quoi le producteur joue-t-il avec ce genre pour en déplacer les règles et dans quelle mesure le récepteur accepte-t-il ou non l'inattendu, la variation? A ce niveau, nous suivons Frédéric François (2004) dans le sens où il se préoccupe précisément de la lecture de textes d'apprentis-scripteurs et du jugement de cohérence que l'on peut leur porter. Son regard sur les textes d'enfants nous invite à bien distinguer d'une part la question de l'intelligibilité d'un texte, de l'autre celle de la réception esthétique d'un texte. Ainsi, l'auteur pose-t-il la question du jugement de cohérence en affirmant que l'analyse textuelle doit certes s'attacher aux ressemblances entre textes mais aussi aux différences, les variantes n'ayant pas du tout le statut d'objets secondaires.

\subsection{Un protocole et un outil d'analyse propres à la confrontation}

Nous avons constitué notre corpus à partir des traces (annotations et commentaires) de l'évaluation de productions textuelles d'élèves de cycle 3 de l'école élémentaire. La situation socio-discursive proposée aux élèves est celle de l'exercice d'écriture ponctuel et non pas celle liée à un projet d'écriture tel que préconisée dans les travaux majeurs en didactique du français ${ }^{(4)}$. La tâche d'écriture retenue

(4) Voir, entre nombreux autres, le $n^{\circ} 36$ de la revue Pratiques sur le travail en projet, $1 \mathrm{e} \mathrm{n}^{\circ} 26$ - 
relève donc des scénarios les plus classiques de l'école : écrire la suite d'un texte narratif $^{(5)}$. Sans entrer ici dans le détail ${ }^{(6)}$, précisons cependant en quoi le texteamorce parait particulièrement adapté à notre objectif. C'est un texte à la fois facile et difficile :

- facile dans le sens où il crée des images relevant des stéréotypes présents dans les textes patrimoniaux et dans les productions cinématographiques : un groupe de compagnons face à un danger de mort. Le texte a effectivement joué son rôle de « déclencheur d'idées » ${ }^{(7)}$;

- difficile car d'une part il met en œuvre un discours du récit complexe tout particulièrement au niveau de l'ordre (anachronie narrative actualisée dans la première phrase), d'autre part, il entremêle des signifiés fournissant des informations paradoxales : aux premières lignes, le lecteur apprend que des hommes sans matériel lourd et sans protection peuvent attraper des requins alors qu'à la fin du texte, le requin devient un animal redoutable.

Notre travail ne se situe pas dans le cadre d'une recherche-action où nous aurions mis en place une collaboration étroite avec un groupe d'enseignants prêts à s'investir dans le projet proposé. Au contraire, notre objectif était d'interagir avec des enseignants forts divers ${ }^{(8)}$ et de leur demander un peu de leur temps pour annoter les textes. C'est pourquoi, l'un des critères de sélection des textes est lié à leur longueur. Nous avons constaté à quel point les enseignants trouvaient assez rapidement fastidieuse leur tâche d'évaluation des textes et cela confirme la pertinence de notre sélection de textes brefs ${ }^{(9)}$. Nous avons par ailleurs choisi des écrits représentatifs des «mondes possibles » construits par l'ensemble des élèves, soit sept textes ${ }^{(10)}$ :

- un monde proche de celui du texte-amorce (une expédition en mer sur un radeau par des savants norvégiens et la présence de requins) et dans lequel le « nageur remarquable » est bien le requin évoqué (parmi les textes retenus, caractéristiques de ceux de Samuel et Alexandre);

- une suite dont le monde s'éloigne de celui du texte-amorce et dans lequel le « nageur remarquable » n'est pas le requin évoqué (parmi les textes retenus, caractéristiques de celui de Valentine);

- un monde proche de celui du texte-amorce mais où le «nageur remarquable » n'est pas le requin évoqué (parmi les textes retenus, caractéristiques de celui de Marvin);

- un monde qui s'éloigne de celui du texte-amorce et dans lequel le « nageur

27 de Repères sur l'apprentissage de l'écriture à l'école élémentaire ou l'ouvrage de Claudine Garcia-Debanc, L'élève et la production d'écrits, collection Didactique des textes, Metz.

(5) Texte amorce en annexe 1.

(6) L'analyse exhaustive se trouve dans notre thèse.

(7) Nous retenons le propos de Caroline Masseron (2005) qui avance l'idée que dans l'exercice de continuation de texte, la commande narrative est plus difficile à satisfaire si le début proposé se trouve en «proximité culturelle» (où l'élève peut s'engager plus facilement dans l'écriture d'un script d'actions routinières) plutôt que s'il consiste en un récit laissant place à l'imagination.

(8) Mais ayant en commun le fait d'avoir suivi une formation (initiale ou continue) en IUFM.

(9) Précisons également que nous avons exclu tous les textes revus; en effet, certains enseignants ayant fait écrire leurs élèves hors de notre présence ont demandé aux élèves de corriger et de réécrire leurs productions. Les textes les plus longs sont justement les textes écrits dans les écoles où nous n'avons pas pu nous rendre.

(10) Annexe 2 
remarquable » est le requin évoqué (parmi les textes retenus, caractéristiques de ceux de Précilia et Camille) ;

- un monde proche de celui du texte-amorce et dans lequel le « nageur remarquable » et un requin dont les attributs ne sont pas ceux du requin du texte initial (parmi les textes retenus, caractéristiques de celui de Zoé).

Nous avons recueilli trois cent vingt cinq textes annotés et commentés ${ }^{(11)}$.

Les chercheurs en didactique qui ont analysé les procédures d'évaluation constatent, d'un commun accord, le poids considérable accordé aux compétences linguistiques locales dans l'évaluation d'une production écrite, en particulier lorsqu'un texte est considéré comme définitif (Halté : 1984 ; Marie-Laure Elalouf : 2005). Nous avons donné à lire aux enseignants des textes à valeur d'écrits définitifs (aucun enseignant d'ailleurs n'a posé la question de la place de ces textes dans une séquence d'apprentissage, peut-être du fait du caractère trop injonctif de notre demande?). Etant consciente du rôle de l'orthographe dans la lisibilité d'un texte, nous ne souhaitions pas voir les enseignants s'y attacher trop longuement. Nous avons donc décidé de corriger les erreurs d'orthographe mais nous n'avons pas modifié la mise en page et la ponctuation choisies par les élèves.

Nous avons commenté les jugements en fonction des trois filtres théoriques présentés ci-dessus, eux-mêmes inscrits dans la dimension englobante de la généricité; nous avons également observé en quoi les enseignants font des commentaires liés à la capacité du texte à aiguiser leur curiosité et donc à montrer un certain pouvoir d'intéressement (Dumortier, 2005), au-delà de la stricte question de la maitrise linguistique. En fonction des deux premiers axes, soient les opérations linguistiques de continuité-discontinuité textuelles et la construction de la cohérence sémantique, nous avons confronté les critères des professeurs pour juger la cohérence du texte. Les outils que mettent à notre disposition les recherches du troisième axe, soit la description des opérations mentales en jeu dans la mise en lien d'éléments textuels, ont permis de différencier les modes de lecture des textes des enseignants. L'ensemble des indices d'une construction-intégration du sens présents dans les jugements de cohérence est pris en compte pour examiner les parcours de lecture des enseignants.

Nous avons systématiquement comptabilisé le nombre de jugements par proposition-énoncé ${ }^{(12)}$ pour chacun des textes soumis à évaluation. Cela a permis de connaître les dimensions qui ont fait l'objet des relevés les plus systématiques. Cet élément a été articulé aux données qualitatives dans le sens où par exemple, une même occurrence a pu être massivement glosée parce qu'elle a été considérée soit comme une réussite soit comme un échec.

L'outil construit pour entreprendre l'analyse prend en compte deux types de données : la catégorie de jugement (selon les axes décrits et commentés supra) et la valeur globale du jugement. Toute évaluation consiste en une recherche de sens qui elle-même se révèle être un jugement de valeur (Gérard : 2002). Nous avons classé les jugements selon deux pôles opposés : les jugements positifs et les jugements négatifs ; dans cette perspective, le classement s'appuie sur les éléments lexicaux et syntaxiques présents dans les écrits des professeurs, particulièrement l'emploi de mélioratifs ou au contraire de dépréciatifs. Nous avons constitué une troisième catégorie qui s'intitule «jugement nuancé », catégorie qui peut sembler

(11) La consigne donnée aux enseignants était la suivante : La suite écrite par l'élève est-elle réussie ou non? Vous pouvez annoter le texte.

(12) Suivant Jean-Michel Adam (2005); cf. 1.1. 
vague : qu'est-ce qu'un jugement nuancé ? La première classe d'expressions linguistiques sur laquelle nous nous sommes appuyée pour noter un propos d'enseignant dans la catégorie «jugement nuancé » est celle des connecteurs argumentatifs ; une seconde classe est prise en compte : celle des modalisateurs. La lecture des jugements montre les éléments considérés comme positifs, les éléments considérés comme négatifs mais aussi en quoi l'opposition montrée relève de la sanction, de l'interrogation ou du doute.

Nous avons choisi d'examiner aussi finement que possible chaque jugement d'enseignant et de mesurer à quel niveau de la cohérence il se situe. Ainsi, nous avons clairement séparé l'analyse de chaque proposition et l'analyse de l'ensemble des appréciations pour un même enseignant. Concrètement, cela veut dire que dans le cadre du premier traitement du corpus, soit les jugements des enseignants à propos d'un même texte d'élève, nous avons traité une remarque classée "positive » et une remarque classée «négative » et écrites par le même enseignant strictement en fonction de notre outil d'analyse, notre but étant de dégager des données quantitatives et qualitatives nous permettant de considérer où se situe la cohérence pour l'ensemble des enseignants (deuxième traitement du corpus). C'est donc dans le troisième traitement du corpus que nous avons repris les jugements d'un même enseignant pour mettre en lien les différentes propositions-énoncés : élément que nous considérons fondamental dans le sens où nous voulions savoir comment les évaluateurs lisent une suite de texte.

Notre méthode a donc consisté à effectuer plusieurs lectures du même corpus ${ }^{(13)}$.

A titre illustratif, nous allons présenter quelques résultats obtenus en fonction des trois niveaux de traitement du corpus : dans un premier temps, nous allons commenter des annotations et remarques qui auront valeur d'exemples représentatifs ${ }^{(14)}$. Dans un second temps, nous mettrons en perspective les conceptions des enseignants concernés afin de caractériser leurs théories implicites. Nous montrerons pour finir que la manière même de lire les textes a à voir avec les conceptions de la cohérence.

\section{Les proto-théories de la cohérence des enseignants}

Depuis la parution de la brochure ministérielle La maîtrise de la langue à l'école ${ }^{(15)}$ en 1992, qui annonçait le double objectif de faire le point sur les travaux des chercheurs dans le domaine de la langue et de fournir aux enseignants des pistes pédagogiques susceptibles de les aider à intégrer les acquis de ces recherches dans leurs pratiques, la cohérence dans ses dimensions pragmatique, sémantique, structurelle est devenue à première vue une notion de base du travail sur le texte. Les derniers programmes pour l'école datant de 2008 précisent à leur tour que l'élève, en fin de cycle 3 de 1'école élémentaire doit rédiger des textes cohérents. Une progression est, de ce point de vue, prescrite du CE2 au CM2 :

CE2 : Rédiger un court texte narratif en veillant à sa cohérence temporelle (temps des verbes) et à sa précision (dans la nomination des personnages et par l'usage d'adjectifs qualificatifs), en évitant les répétitions par l'usage de synonymes, et

(13) Voir le tableau récapitulatif des axes d'analyse en annexe 3. Tous ne seront pas commentés dans le cadre de cet article.

(14) Les enseignants ont exprimé 1744 jugements à propos des sept textes soumis à leur lecture.

(15) Ministère de l'Education nationale et de la Culture (1992), La maîtrise de la langue à l'école, Paris : Savoir Livre CNDP. 
en respectant les contraintes syntaxiques et orthographiques ainsi que la ponctuation.

CM1 : Rédiger des textes courts de différents types (récits, descriptions, portraits) en veillant à leur cohérence, à leur précision (pronoms, mots de liaison, relations temporelles en particulier) et en évitant les répétitions.

CM2 : Maîtriser la cohérence des temps dans un récit d'une dizaine de lignes.

Rédiger différents types de textes d'au moins deux paragraphes en veillant à leur cohérence, en évitant les répétitions, et en respectant les contraintes syntaxiques et orthographiques ainsi que la ponctuation. (Programmes $2008: 35$ ).

Cette prescription récente n'est accompagnée d'aucune piste bibliographique ni d'aucun commentaire à visée théorique ou pratique ${ }^{(16)}$. Cependant elle implique (1) que les enseignants doivent être capables de juger la cohérence d'un texte (2) qu'ils puissent en dégager pour les élèves d'éventuels besoins langagiers (3) qu'ils sachent mettre en place des dispositifs d'aide à l'élaboration de la cohérence. Ainsi, la cohérence apparait comme un objet incontournable dans le cadre de l'enseignement / apprentissage du français.

Les enseignants qui ont évalué les textes ont montré leur propre exigence interprétative, que ce soit au niveau des effets de texte ou au niveau des effets de monde, dimensions que nous examinons maintenant en fonction des axes d'analyse retenus.

\subsection{Première lecture du corpus : annotations d'un même texte par le groupe d'enseignants}

\subsubsection{Continuité - discontinuité textuelle}

De l'analyse textuelle des discours, nous avons retenu qu'un texte est un matériau verbal qui s'actualise si certaines unités s'articulent et se lient entre elles (Adam, 2005). Dans l'espace imparti à cet article, nous allons porter notre attention sur quelques annotations et remarques à propos de l'emploi des temps et des

anaphores ${ }^{(17)}$. Nous nous arrêtons sur ces faits de texte parce qu'ils représentent deux des notions pivots de la didactisation de la linguistique textuelle depuis une trentaine d'années. Aussi - et en conséquence - les enseignants ont très régulièrement évalué ces faits dans les copies des élèves. Nous comptons en effet une moyenne de vingt-neuf jugements par texte à propos de l'emploi des temps et de dix-sept jugements par texte en ce qui concerne les anaphores.

L'emploi des temps est l'un des rares pôles où des jugements positifs sont exprimés en nombre ${ }^{(18)}$. Le texte amorce articule de façon homogène le passé simple et l'imparfait, ce qui provoque une mise en relief clairement identifiable. L'extrait est donc représentatif des textes donnés aux apprenants dans le cadre de 1'approche en classe du premier plan et de l'arrière-plan dans le récit ${ }^{(19)}$.

(16) Les programmes précédents étaient en revanche accompagnés de fascicules explicatifs et illustratifs.

(17) Pour chaque fait analysé, nous choisissons de nous limiter à la présentation de quelques catégories de remarques qui nous semblent significatives des conceptions des enseignants concernés et des questions qu'elles peuvent poser dans le cadre de la didactisation des savoirs savants et de la formation des enseignants.

(18) Généralement, ne sont explicitement annotés que les faits considérés comme des erreurs, des manques, des approximations.

(19) Et ce dès l'école élémentaire ; voir par exemple Mon Manuel de Français, Retz, 2006. 
Voici par ailleurs quelques segments interrogés et/ou disqualifiés et que nous commentons dans le sens où nous essayons de comprendre à la fois le choix d'écriture de l'élève et la sanction de l'enseignant:

Sanction de l'imparfait dans le discours citant:

Extrait (1) : Knut se fit manger par le requin, les autres criaient « au secours, au secours » mais malheureusement à ce moment-là ils étaient en plein milieu de l'océan. (Camille)

Exemples de remarques ${ }^{(20)}$ : «erreur de temps : imparfait au lieu de PS»; «Temps verbal inadéquat »; " usage des temps (imparfait / PS) 》; «confusion des temps»

Comment la correction apportée par certains enseignants, soit la modification de «criaient» en «crièrent» se justifie-t-elle ? Nous pensons que nous sommes face à une question liée à la «vision du procès », que nous opposons à l'idée de réalité concrète : le choix de l'imparfait ou du passé simple modifie non pas le contenu propositionnel mais la manière dont le sujet parlant considère le procès : au passé simple, l'action est mise en relief, à l'imparfait, elle devient « toile de fond ». Dans ce cadre, par l'emploi du passé simple, les enseignants concernés bornent le fait de crier et estiment donc que le seul élément textuel que l'on peut voir en train de se vivre est le fait que le groupe est en plein milieu de l'océan, effet provoqué par l'emploi de l'imparfait. Choisir l'imparfait pour «crier » est-il problématique? Pour répondre à cette question, reprenons l'organisation des temps verbaux sur 1'ensemble du texte de Camille ${ }^{(21)}$ : le texte commence au passé simple pour parler de l'événement central : Knut se fait manger par le requin. Après les deux propositions-énoncés [é3]-[é4] à l'imparfait, le texte se poursuit en [é5] sous forme dialoguée, avec immédiatement un verbe au présent de l'impératif, dans une syntaxe impérative qui est en elle-même un marqueur de force illocutionnaire : il s'agit de donner un ordre pour intimer l'autre d'agir ([é5] - prenez les baïonnettes, dit le capitaine).

Quelles valeurs donner aux deux imparfaits encadrés par les procès faisant avancer l'action?

L'élève le dit lui-même dans l'écriture de son texte : le fait de crier n'est pas considéré comme un événement à privilégier (crier ou non ne change rien puisqu'ils sont au milieu de l'océan) mais c'est l'état de fait, l'à-côté du récit qui va donner toute leur force aux paroles du capitaine.

Ainsi, nous obtenons schématiquement : actions (en [é1]-[é2]); commentaires (en [é3]-[é4]); actions à partir de [é5].

Sanction de l'imparfait en position seconde dans une phrase coordonnée où le premier verbe est au passé simple

Extrait (2) : Jarod prit un bâton et tapait sur le requin. (Samuel)

Exemples de remarques ${ }^{(22)}$ : «une petite erreur de temps»; «quelques bonnes expressions mais un mauvais emploi des temps »; «confusion imparfait / passé simple "; "cohérence verbale non respectée ».

(20) Nous comptons 14 jugements négatifs. Les enseignants ont généralement barré 1'occurrence et 1 'ont remplacée par «crièrent».

(21) En annexe 2.

(22) Nous comptons 15 jugements négatifs. 
En quoi l'imparfait choisi par Samuel est-il une « erreur »? Elargissons 1'empan textuel afin de relire les cotextes de gauche et de droite :

[é2] Le requin essaya de le mordre [é3] mais en vain. [é4] Knut sauta sur le radeau.

[é5] Jarod prit un bâton [é6] et tapait sur le requin. [é7] Mais celui-ci cassa le bâton.

Si le passé simple est le temps préférentiel du marquage de la succession des actions, taper devrait revêtir le passé simple selon la règle d'avancement des actions dans une série au passé simple : Jarod prit d'abord le bâton et tapa le requin. S'ensuit dans la chronologie la réaction du requin avec le procès suivant : " cassa ». Donc, du point de vue d'une sémantique temporelle, il faut effectivement choisir le passé simple. Mais le passé simple est-il le seul temps capable de montrer qu'un procès est postérieur à un autre dans un récit ? Un certain nombre d'exemples attestés montre la possibilité d'un imparfait narratif, par exemple :

La clef tourna dans la serrure. Monsieur Chabot retirait son pardessus qu'il accrochait à la porte d'entrée, pénétrait dans la cuisine et s'installait dans son fauteuil d'osier (Simenon, La danseuse du Gai-Moulin, cité par L. de Saussure et Sthioul 2005).

Dans ce cas, le procès perd sa perspective sécante et prend une valeur identique à celle du passé simple.

Nous venons d'apparier [é6] au cotexte de gauche, soit [é5].

Qu'en est-il si, au-delà de la barrière du point terminant la phrase graphique, nous apparions [é6] et [é7]?

[é5] Jarod prit un bâton [é6] et tapait sur le requin. [é7] Mais celui-ci cassa le bâton.

Cette lecture se justifie, selon nous, du fait de la présence en initiale de [é7] du connecteur « Mais » qui annonce une opposition tranchée et donc un lien de contraste fort (Weinrich : 1989) entre les deux propositions [é6] et [é7]. Cette lecture implique d'attribuer une nouvelle valeur à l'imparfait, qui retrouve alors sa perspective sécante. Les deux procès en [é6] et [é7] ne se succèdent pas, mais connaissent un point d'intersection : c'est pendant qu'il tapait sur le requin que ce dernier cassa le bâton ${ }^{(23)}$.

En conclusion, les remarques des enseignants à propos de l'imparfait jugé erroné nous ont amenée à constater que tout comme les connecteurs ou les déictiques, les temps verbaux sont des marques relationnelles, dans le sens où l'on peut tenter de reconstruire la procédure que met en place le destinataire pour interpréter le temps verbal concerné dans un contexte donné. Nous ne pouvons savoir quelle contrainte linguistique, contextuelle, cognitive a mené Samuel à choisir l'imparfait. En revanche, nous pouvons discuter le fait que sa proposition se réfère à des possibles linguistiques et textuels en termes d'emploi des temps.

Sanction du présent dans une section de texte en énonciation récit :

$$
\text { Extrait (3) }{ }^{(24)} \text { : Et ils se marient (Valentine) }
$$

(23) C'est cette interprétation que nous avons retenue dans notre primo-lecture du texte de Samuel où nous avons donné à l'imparfait une valeur descriptive et itérative, permettant une vision analytique de l'événement.

(24) Nous comptons 14 jugements négatifs. 
Exemples de remarques : "Maîtrise des temps. Sauf à la dernière phrase 》; « revoir le temps »; "attention au temps utilisé»; "incohérence : cohérence des temps verbaux: les temps du récit».

Les enseignants concernés (soit 35\% des évaluateurs du texte de Valentine) ont systématiquement et rigoureusement apparié le conte et le passé simple. Il est en effet indéniable que le passé simple est le temps préférentiel du mode d'énonciation récit. L'emploi par l'élève de la formule rituelle de clôture du conte renforce l'attente d'un passé simple. Mais peut-on pour autant affirmer que le présent employé en [é11] est fautif?

Voyons si l'effet de texte s'avère différent lorsque l'on emploie le passé simple :

Arielle dit : je te donnerai une queue.

Et ils se marièrent.

Il semble que l'effet sur le récepteur ne change pas fondamentalement, car si problème il y a, il ne se situe ni en [é10] ni en [é11], mais dans le blanc qui les sépare.

On peut avancer malgré tout deux hypothèses à propos du présent en [é10], l'une pour affirmer qu'il existe cependant une nuance interprétative entre la version au présent et la version au passé simple, l'autre pour évoquer ce qui nous semble être une contrainte cotextuelle d'emploi du présent. Nous avons noté qu'aucun enseignant n'évoque ces points dans les annotations du texte de Valentine.

La place même de l'occurrence «se marient», en toute fin du texte, nous invite à revenir sur l'une des fonctions du passé simple : faire avancer le récit, permettre aux actions de se succéder, or, ici, il n'y a plus nécessité d'avancer, l'histoire est finie. En revanche, dans un récit, le présent plonge le récepteur au cœur de l'action. Dans le texte de Valentine, la résolution n'est pas anodine : par le motif du mariage, elle évoque le bonheur, l'amour, l'éternité. Ainsi

le temps roi est le présent de narration qui donne au lecteur l'impression qu'il assiste à l'action et par là lui imprime des émotions plus vives. (Mónica Morillo Vicente $2004: 10)$.

Par ailleurs, nous pensons que le choix (ou la contrainte) de l'emploi du présent sont liés à sa place dans l'économie générale du texte : l'occurrence se situe juste après le verbe dire dont on connait l'ambiguïté formelle à la troisième personne pour laquelle l'on ne peut pas savoir s'il s'agit du présent ou du passé simple; Valentine l'emploie à quatre reprises dans le discours attributif. Il est, dans ce contexte, impossible de déterminer quelle valeur possède exactement le présent en [é11].

Les anaphores ont donné lieu pour les sept textes soumis à évaluation à cent dix sept remarques dont cent sous la forme d'un jugement négatif. Nous n'allons pas présenter des occurrences qui remettent en question sans conteste l'intelligibilité du texte mais des annotations qui nous semblent sources de conflits interprétatifs et donc intéressantes dans le cadre de notre recherche de la définition de la cohérence par les enseignants. Dans les deux exemples que nous présentons, la sanction est liée à un problème d'accessibilité référentielle :

Extrait (4) : [é5] Jarod prit un bâton [é6] et tapait sur le requin. [é7] Mais celuici cassa le bâton.

Exemples de remarques ${ }^{(25)}$ : «l'utilisation du pronom celui-ci est impré-

(25) Nous comptons 5 remarques négatives. 
cise »; "Reprise utilisée pour Jarod ou le requin?»; «de quel personnage parles-tu?»

Nous nous trouvons dans une situation tout à fait intéressante dans le sens où une reprise comme «celui-ci » fait partie des formes anaphoriques spécialisées dans le contrôle des risques d'ambiguïté (Charolles : 1995).

Pourquoi plusieurs enseignants considèrent-ils l'identification du référent comme peu évidente? Il nous semble qu'il y a une double raison : d'une part, le fait que la récupération pour l'interprétation d'un référent s'appuie sur un contrôle linguistique, or, «Jarod» et « requin » sont deux noms au masculin singulier : il n'y a donc pas de moyens grammaticaux de choisir le «bon antécédent». D'autre part, le fait que le procès en jeu, soit « casser », accepte comme agent aussi bien un individu ayant le trait /humain/ qu'un individu ayant le trait/animal/ : Jarod peut taper et casser le bâton; le requin peut réagir aux coups et casser le bâton.

L'emploi de « celui-ci » par l'élève est-il, dans ce cadre, un choix pertinent ? Pour nous, la réponse est positive. En choisissant «celui-ci » et pas simplement « il », l'élève oriente l'attention du lecteur sur le référent « requin ». En effet, réécrivons l'extrait concerné en substituant « il » à « celui-ci »:

[é5a] Jarod prit un bâton [é6] et tapait sur le requin. [é7] Mais il cassa le bâton.

Dans ce cas, le pronom « il» en position sujet invite à considérer que l'orientation du discours reste identique : le focus reste le même et est totalement accessible. Il n'y a pas de changement d'agent. Si l'on passe du rôle de lecteur à celui de producteur de textes, on peut d'ailleurs noter que lorsque l'on ne souhaite pas provoquer de changement d'orientation dans la chaîne référentielle, on emploie habituellement la reprise la plus classique, c'est-à-dire le pronom « il ». Or, ce n'est pas ce que fait Samuel. En choisissant «celui-ci», il invite le lecteur à se détourner de «Jarod » et à considérer « le requin » comme référentiellement saillant. Ainsi, l'anaphore « celui-ci » remet en question l'organisation référentielle à laquelle on peut s'attendre «par défaut».

Pour conclure sur cette occurrence, il nous semble que le doute des enseignants concernés remet en question une intuition linguistique pertinente de l'élève. Ce dernier montre en effet la capacité à gérer de façon subtile l'avancée de l'information, même si, comme l'affirme Michel Charolles à propos des jeunes scripteurs :

la maîtrise des principes (hautement intelligents, on l'a vu) gouvernant le fonctionnement des formes anaphoriques ne passe certainement pas par une connaissance réflexive. (1995: 111)

Extrait (5): L'un des cinq compagnons prit une corde et l'attache sur le radeau. La lance et Knut l'attrape. (Alexandre)

Exemples de remarques ${ }^{(26)}$ : «la pronominalisation n'est pas maîtrisée 》; "Le "l"” de la première phrase n'a pas de référent explicite. On ne sait pas si les compagnons attachent la corde, Knut ou le requin"; "à quoi cela renvoie?»

Regardons le cotexte de droite :

[é1]L'un des cinq compagnons prit une corde [é2] et l'attache sur le radeau. [é3] La lance [é4] et Knut l'attrape.

(26) Nous comptons 13 remarques négatives. 
La prise en compte de l'environnement référentiel élargi permet de lever l'ambiguïté et ce dès [é3], où le genre et le nombre du pronom personnel suffisent à (r)établir la co-référence. Or, le pronom personnel est situé après un point. Les études consacrées à la gestion de la ponctuation dans la construction de la cohérence d'un texte ont montré que les signes ponctuatifs jouent au moins deux rôles dans la compréhension : 1) ils établissent des liens entre unités. Dans cette perspective, une différence de traitement est sensible chez le lecteur : en présence d'une virgule, il met en œuvre un calcul d'inférence pour lier les parties séparées par cette marque. En revanche, le point est considéré comme une marque de rupture. 2) La fin d'une phrase est le lieu privilégié de l'intégration sémantique : le point devient dans ce cadre le signal fort du traitement de l'information à disposition (Passerault : 1991 ; Roy, Gaonac'h, Fayol : 2002). Ces deux fonctions du point ont sans doute à voir avec la manière dont les évaluateurs sanctionnent le segment textuel composé de [é1]-[é2]. Ils semblent avoir focalisé leur attention sur une plausibilité locale, en s'en tenant exclusivement au cotexte de gauche.

\subsubsection{Cohérence sémantique}

De la sémantique textuelle, nous avons retenu que le lecteur construit une signification dans une dynamique fondée sur une sélection thématique qu'il réalise à partir des scénarios intertextuels qu'il connait. Dans ce cadre, en quoi les mots et leurs entours, choisis par les élèves, ont ouvert un ou des monde(s) que les évaluateurs ont considéré en cohérence ou non avec l'idée qu'ils se forgeaient du texte à produire ? Nous allons observer deux exemples de tensions entre topics et isotopies :

\section{Dans le texte d'Alexandre}

Nous avons recherché dans les commentaires quels sont les marqueurs de topic et plus généralement les mots clefs qui ont été saillants pour les lecteurs. Nous avons constaté que les moyens de résolution du problème sont discutés par les enseignants. Pour certains, il y a co-occurrence de certains indices qui renvoient au même topic « récit d'aventure », ce qui est considéré plutôt comme une réussite :

«Alexandre a écrit un texte d'aventure (vocabulaire : corde, requin, radeau, naviguer,...) L'action progresse, il y a du suspens »; "On ne sait rien des rames et de la corde, mais rien de contredit leur usage!».

Pour ces enseignants, le groupe de lexèmes (corde, requin, rames, radeau, naviguer) semble en adéquation avec la représentation du texte qu'ils ont construite et qui pourrait prendre le titre de «Aventure sur un radeau en plein océan ». Les éléments nouveaux proposés par l'élève (la corde, les rames) ne remettent pas en cause l'uniformité du récit.

Ce point de vue n'est pas partagé par tous les enseignants, par exemple :

"L'élève a donné du matériel à l'équipage que dans le début du texte[sic], ils $n$ 'ont rien dans le radeau $\Rightarrow$ compréhension difficile du récit»; un évaluateur souligne « corde » et "rame », renvoie par un fléchage à un extrait du texteamorce (en se laissant entraîner par les courants) et note : "problème de corde! Problème de rame! ».

Nous constatons que pour les mêmes occurrences, les avis sont contradictoires : pour certains enseignants, il semble s'agir de respecter au plus près les éléments contextualisés dans le texte-amorce. Les parcours interprétatifs s'actualisent par 
l'inférence consistant à activer l'idée que les compagnons «n'ont rien dans le radeau » alors que le texte-amorce ne le dit pas. Les actants nouveaux (la corde et les rames) sont considérés comme contradictoires, alors que pour un enseignant, ces objets ne font pas perdre à l'expérience des compagnons son authenticité.

Pourquoi peut-on admettre « corde » et « rame»?

Peut-être en analysant la tension entre un topic construit à partir des mots clefs donnés dans le texte de contextualisation de l'extrait et les mots introduits par Alexandre; dans le texte de contextualisation, c'est le pan de phrase « sans moteur et sans voiles, en se laissant entraîner par les courants » qui active un dictionnaire de base du récepteur : en effet, on peut admettre que, sans nécessité ici d'un contexte ou d'un cotexte très développé, l'on peut activer des propriétés sémantiques actualisant le topic « moyen de navigation sommaire».

Cependant, les enseignants semblent avoir traité de façon différenciée les lexèmes « rame» et « corde» : pour deux d'entre eux, le trait saillant des deux mots est /non modernité/ et dans ce cadre, il s'agit de compléter l'isotopie autour du champ lexical renvoyant à des indices co-occurrents du topic « moyen de navigation sommaire ». En revanche, pour les deux autres, ce sont les traits /manque//absence/ /exclusion/ actualisés par le lexème "sans » répété deux fois qui semblent saillants et qui mènent à la sanction des occurrences « corde » et « rame» dans le texte d'Alexandre : sur le radeau, il n'y a rien.

Ces annotations sont particulièrement instructives quant à la recherche des liens entre topic et isotopie : en effet, elles montrent que dans un contexte qui n'est, à première vue, pas particulièrement ambigu, les scénarios textuels activés sont distincts. Mais, s'ils se différencient en fonction des encyclopédies personnelles, ils s'appuient aussi crucialement sur les instructions données par le texte.

\section{Dans le texte de Précilia}

La part prise par le requin dans la dynamique des actions donne lieu à quatorze remarques. Les enseignants concernés attribuent sans aucun doute le rôle d'opposant au requin. Mais au-delà de la construction sémiotique, ils posent différents points de vue sur le monde narratif construit par le texte.

Un enseignant valorise le fait que l'isotopie liée au danger que représente le requin est conservée : "Le côté menaçant de l'ombre est réexploité ".

En revanche, neuf remarques interrogent différents éléments du monde textuel proposé :

Les enseignants interviennent à propos de l'association sémantique « requin/ tomber » et de l'inférence qu'elle implique : si le requin ne tombe pas, c'est donc qu'il est sur/dans (?) le radeau. Soit ils montrent leur étonnement, par un soulignement de la proposition [é2] ([é2] mais le requin ne tombait pas.) suivi d'un point d'interrogation, soit ils glosent, par exemple :

"Sait-il ce qu'est un requin? "; " pourquoi le requin devrait-il tomber? » ; "Ils atteignirent l'embarcation est compris comme " monter dans... " car l'enfant dit : le requin ne tombait pas ».

Un enseignant entre de plain-pied dans le monde construit par le texte et ajoute un bordé au radeau en écrivant «bateau » au lieu de " radeau ». Ainsi pouvonsnous lire: «Il semble qu'il manque le moment qui suit le temps où Knut et le requin atteignent l'embarcation. Le moment où les deux personnages montent sur le bateau n'est pas raconté. » (souligné par nous). 
Au final, pour huit enseignants, l'événement raconté n'est pas compatible avec leurs propres schémas conceptuels. Le neuvième enseignant quant à lui, reconstruit un script pour tenter de réduire le désaccord entre ce qu'il lit et sa propre construction du monde du texte amorce : il rétablit un rapport d'intériorité dans l'espace (bateau au lieu de radeau ). Dans cette perspective, sa remarque n'est pas une remise en question de la cohérence du monde proposé par l'élève. Cet enseignant semble donc commettre ce que des psychologues appellent une erreur reconstructive ( $c f .1 .1)$.

\subsubsection{Pouvoir d'intéressement}

Nous n'avons pas mené d'entretiens avec les évaluateurs, il peut donc paraître assez vain de vouloir connaître leur plaisir/déplaisir ressentis à la lecture des textes des élèves. Cependant, notre hypothèse de départ était que même dans une évaluation écrite fondée sur la réussite d'un texte en regard d'une consigne, les enseignants feraient quelques remarques exprimant leur position par rapport non pas au strict respect de la consigne mais à l' effet produit. Nous avons effectivement relevé des annotations marquant un plaisir ou un déplaisir mais elles sont relativement peu nombreuses (quarante-six sur l'ensemble des textes). Nous présentons dans un tableau quelques-unes des appréciations pour chaque texte :

\begin{tabular}{|c|c|c|}
\hline Texte de : & Jugements positifs & Jugements négatifs ou nuancés \\
\hline Alexandre & $\begin{array}{l}\text { - Histoire très intéressante } \\
\text { - Imaginaire }++ \\
\text { - L'idée du texte est recevable }\end{array}$ & $\begin{array}{l}\text { - Dialogues peu pertinents } \\
\text { - Fin peu intéressante } \\
\text { - Langage trop familier } \\
\text { - Par la dernière phrase (« vraiment } \\
\text { beau le radeau ! »), on sent la lassitude } \\
\text { de l'élève. Il semble se « débarrasser » } \\
\text { de la tâche d'écriture }\end{array}$ \\
\hline Précilia & $\begin{array}{l}\text { - Récit qui a une fin et une belle fin! } \\
\text { HAPPY END! } \\
\text { - C'est drôle! } \\
\text { - C'est intéressant de faire participer } \\
\text { tous les personnages }\end{array}$ & $\begin{array}{l}\text { - Pas terrible } \\
\text { - Peu palpitant ; manque d'imagination } \\
\text { - Manque de détails }\end{array}$ \\
\hline Valentine & $\begin{array}{l}\text { - Très jolie fin } \\
\text { - Très belle idée } \\
\text { - L'idée de la sirène est intéressante }\end{array}$ & $\begin{array}{l}\text { - Dialogue pauvre } \\
\text { - Cette suite est fantaisiste } \\
\text { - Histoire trop simple }\end{array}$ \\
\hline Samuel & $\begin{array}{l}\text { - J'aime bien } \\
\text { - Cette histoire me semble très bien } \\
\text { écrite } \\
\text { - expressions intéressantes }\end{array}$ & $\begin{array}{l}\text { - Texte un peu court } \\
\text { - Dialogue pauvre }\end{array}$ \\
\hline Camille & $\begin{array}{l}\text { - De bonnes idées! } \\
\text { - Texte très vivant } \\
\text { - Une suite digne de certains films }\end{array}$ & $\begin{array}{l}\text { - Ça bouillonne d'idées mais il faut ap- } \\
\text { prendre à les structurer dans l'écrit }\end{array}$ \\
\hline Marvin & $\varnothing$ & $\begin{array}{l}\text { - Imaginaire non retranscrit si existe } \\
\text { - Les faits sont inintéressants }\end{array}$ \\
\hline Zoé & $\begin{array}{l}\text { - Un requin qui parle ? Pourquoi } \\
\text { pas? Après tout nous sommes dans } \\
\text { une fiction, même si le début de } \\
\text { l'histoire ne laisse pas présager cela }\end{array}$ & $\begin{array}{l}\text { - l'histoire est peu réaliste } \\
\text { - situation de dialogue peu probable }\end{array}$ \\
\hline
\end{tabular}


Cet échantillon permet de constater la divergence des points de vue sur les textes d'élèves. Par exemple, les enseignants expriment des jugements liés au contenu, valorisant des univers pourtant en rupture totale avec le texte amorce (texte de Valentine), valorisant des dynamiques d'actions (texte de Camille), disqualifiant une clôture en inférant l'état psychologique de l'élève (texte d'Alexandre) et des jugements liés à l'expression (valorisée par exemple dans le texte de Samuel et disqualifiée dans le texte de Camille).

Comme nous l'avons précisé plus haut, les quelques exemples d'annotations et de remarques que nous avons sélectionnées ne couvrent en aucun cas l'ensemble des faits de texte que nous avons observés mais sont cependant assez représentatifs des jugements portés sur les textes. Après avoir confronté des remarques du groupe d'évaluateurs à propos d'un même segment textuel, nous allons présenter notre seconde lecture du corpus, c'est-à-dire considérer en quoi les jugements nous permettent de proposer une cartographie des conceptions de la cohérence des enseignants à propos des faits discutés en 2.1. Nous élargissons nos commentaires à l'ensemble des annotations.

\subsection{Deuxième lecture du corpus : les proto-théories de la cohérence des enseignants}

\subsubsection{Une vision plutôt restrictive des liages sémantiques (cohésion verbale) et référentiel (anaphores)}

Qu'ils parlent de «concordance des temps», de « cohérence temporelle » ou d'« emploi des temps », les enseignants semblent peu favorables à la variation énonciative. Lorsqu'ils interrogent ou sanctionnent un temps verbal, il s'agit dans la majorité des cas d'un appel à une reprise systématique des temps du texteamorce, qui, nous le rappelons, s'inscrit dans une narration disjointe, employant les deux temps de base que sont le passé simple et l'imparfait.

Les annotations montrent que de façon majoritaire, toute alternance temporelle est interprétée comme une rupture. Un seul commentaire sur l'ensemble tente d'analyser la «logique temporelle » de l'élève en donnant au passage du passé simple au présent le sens d'une mise en valeur de l'épisode. Les questions que cet état de fait soulève sont très complexes. Une question linguistique d'abord : en quoi les alternances sont-elles aléatoires ou en quoi relèvent-elles effectivement d'une logique temporelle? Une question didactique ensuite, liée à l'évaluation dans le cadre scolaire : dans quels cas les formes considérées comme des ruptures par les enseignants sont-elles des erreurs d'apprentis ? Dans quels cas montrent-elles au contraire une maîtrise de leur dimension textuelle ? Une réponse est apportée par les recherches sur lesquelles nous nous sommes appuyée pour analyser les choix temporels des élèves et qui nous ont permis de partager le point de vue de Facques (2002), à savoir : analyser les décisions d'un auteur consiste à tenir compte à la fois du caractère éminemment hétérogène des textes et des contraintes liées au choix d'un genre textuel. Facques invite à dépasser deux écueils possibles à propos de 1'analyse des alternances temporelles : considérer ces dernières uniquement comme des particularismes idiosyncrasiques ou au contraire ne les considérer qu'au regard des valeurs de temps en système. Ce point de vue est partagé par Dolz (1993) qui étudie l'hétérogénéité temporelle en partant de l'idée que tout texte, du fait de son inscription dans un genre de référence, s' appuie sur une « base temporelle » qui constitue l'ancrage à partir duquel peuvent s'actualiser des déri- 
vations plus ou moins attendues et pouvant parfois s'apparenter à des ruptures temporelles. Nous retrouvons la situation vécue par les élèves : la base temporelle était imposée par le texte-amorce et chacun a géré cette contrainte en créant des effets qui ont été jugés par les enseignants.

Proto-théorie des enseignants : la cohésion verbale se caractérise de façon majoritaire par le critère d'homogénéité énonciative : un texte bien formé du point de vue des temps verbaux est un texte régulier et «limpide» (Combettes, 1995).

Les anaphores représentent une unité textuelle annotée de façon tout à fait déséquilibrée en fonction des textes d'élèves, du fait de la part peu importante des phénomènes de reprise au sein de certains écrits mais aussi parce que les enseignants n'ont dans l'ensemble pas proposé d'appréciations explicites lorsqu'ils ont considéré que les faits concernés montraient une compétence textuelle (nous comptons neuf jugements positifs sur les cent dix-sept relevés). Au final, trois des textes d'élèves regroupent $90 \%$ des remarques. Les conceptions de la cohésion nominale peuvent s'inférer à partir de deux types de remarques : des demandes de traitement d'une ambiguïté référentielle mettant en jeu une approche linguistique ; des demandes de traitement d'une ambiguïté référentielle qui entraine une discussion sur des dimensions discursive et mémorielle du phénomène de reprise. Les premières s'appuient essentiellement sur des critères de proximité textuelle et de continuité linguistique au niveau du genre et du nombre. Les secondes, plus complexes, articulent les fonctions textuelle, sémantique et pragmatico-cognitive de l'anaphore.

Certains enseignants se montrent parfois très exigeants dans leur souhait de clarification. Par exemple, dans l'extrait suivant, la demande d'explicitation du référent du pronom ils sanctionne à notre sens une performance textuelle de l'élève qui gère le discours citant de façon assez élaborée :

- Où est le requin? dit un spécialiste.

- Il est là! crient les enfants en chour.

- On s'en occupe! dirent les autres spécialistes.

- Pouvez-vous nous ramener ?dirent-ils tous en chœur. (Précilia)

Certains enseignants ne prennent manifestement pas le cotexte de droite comme lieu de résolution de l'anaphore ( $L$ 'un des cinq compagnons prit une corde

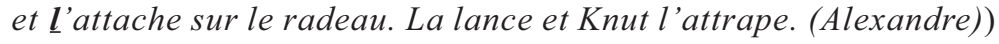

Nous avons également relevé un problème au niveau du rôle attribué à des anaphores spécialisées. (Jarod prit un bâton et tapait sur le requin. Mais celui-ci cassa le bâton. (Samuel).)

Proto-théorie des enseignants : la cohésion nominale est considérée comme un élément de texture qui établit un lien étroit entre un référent et sa reprise. La conception des enseignants apparaît majoritairement « antécédentiste » (Apothéloz, 1995) dans le sens où seul le cotexte immédiat et de gauche est pris en compte comme lieu de résolution de l'anaphore. La lecture-évaluation de textes d'apprentis semble évacuer la question de l'environnement discursif dans l'interprétation des anaphores. 


\subsubsection{Une vision singulière des effets de monde ; l'intelligibilité plus que la réception esthétique}

Aucune suite produite ne fait 1'unanimité quant à sa cohérence du point de vue des choix narratifs menant à la résolution : pour six textes, les avis sont divergents et pour le septième, écrit par Samuel, ils sont nuancés. Au-delà de l'intelligibilité des textes, leur pouvoir d'intéressement est également évalué de façon très variable.

Du point de vue de l'intelligibilité, il y a pour certains enseignants un problème de cohérence lié au choix par les élève de certains objets. En effet, des élèves ont introduit des objets susceptibles de rendre cohérente l'histoire en vue de sa résolution : il s'agit d'objets investis de valeurs objectives, tels une corde, des rames, des baïonnettes, des balles, un bâton. Cette énumération appelle les commentaires des enseignants sur le respect du monde construit par le texte-amorce. Au travers de ce jugement, que disent les professeurs ? Pour aucun des objets cités, nous ne lisons un avis univoque. Un examen sémantique montre que les lexèmes ne sont pas considérés de façon lexicale, c'est-à-dire comme des organisations sémiques virtuelles, mais de façon textuelle et co(n)textuelle, donc comme des éléments d'un champ figuratif partie prenante du parcours interprétatif. Mais, les distorsions apparaissent selon le co(n)texte inféré et/ou activé. Par exemple, la corde et la rame sont acceptées ("on ne sait rien des rames et de la corde mais rien ne contredit leur usage ») et refusées ( "problème de corde! Problème de rame! »). Le bâton, objet primitif non manufacturé, accepté implicitement par la majorité des évaluateurs est cependant remis en question par deux enseignants. Et de façon assez paradoxale, quatre enseignants seulement interrogent explicitement l'orientation du texte de Camille vers le topic navire de guerre, alors que le texte met en jeu une série d'expressions lexicales non ambiguës de ce point de vue.

Nous avons émis l'hypothèse que les enseignants exprimeraient leurs ressentis en fonction de leur appréciation des efforts cognitifs et imaginatifs attribués aux élèves en tant que " producteurs d'effets de sens » (François 2004 : 136). Nous relevons effectivement des avis qui ont trait explicitement au plaisir ou au déplaisir que les enseignants ont pris à découvrir les textes. Les remarques, de ce point de vue, se situent bien dans le cadre définitionnel du pouvoir d'intéressement décrit par Dumortier (2005), c'est-à-dire qu'elles sont fondées sur des arguments prenant appui sur des lieux de la création narrative dans les deux directions que sont le contenu et l'expression. Cependant, ces remarques explicites sont peu nombreuses et présentent une variation numérique importante d'un texte à l'autre (de deux à dix-huit jugements).

Proto-théorie : Pour certains enseignants, la résolution doit montrer une homogénéité thématique et respecter la règle du vraisemblable. Ce dernier doit prendre appui sur le savoir humain acquis ou considéré comme «vrai » dans le cadre de leur propre encyclopédie. Pour d'autres enseignants (bien moins nombreux) le texte peut s'affranchir de la stricte répétition et «jouer » de l'interaction du genre de référence avec d'autres genres.

\subsection{Troisième lecture du corpus : les parcours de lecture}

La relative banalité de l'exercice de continuation textuelle rend a priori évident le fait de fonder l'évaluation sur l'examen de la compétence des élèves à gérer des règles de reprise. C'est effectivement ce que nous constatons en observant le corpus. Cependant, l'analyse attentive des annotations met au jour le phénomène sui- 
vant : les appréciations se distribuent de façon différenciée, selon que l'enseignant annote le texte ou bien propose une remarque hors du texte et après la lecture complète de la production de l'élève. Nous souhaitons préciser cet état de fait.

Pour obtenir une image des modes de lecture des enseignants, nous avons croisé deux types de données : le moment du jugement et l'unité textuelle considérée. Deux moments sont repérables : le jugement en cours de lecture et le jugement après lecture. Deux unités textuelles sont prises en compte : soit uniquement le texte produit, soit le continuum entre le texte-amorce et le texte produit. L'application du dispositif d'analyse a permis de repérer trois modes de lecture dominants :

1- l'évaluation de la suite uniquement d'un point de vue interne en cours de lecture comme dans le commentaire final;

2-1'évaluation de la suite d'un point de vue interne en cours de lecture et dans le continuum avec le texte-amorce dans le commentaire final ;

3- l'évaluation de la suite dans le continuum avec le texte-amorce en cours de lecture et dans le commentaire final.

Nous avons récapitulé les résultats qui se manifestent à la croisée de ces données pour l'ensemble des textes et pour chaque texte évalué. Nous présentons cidessous les résultats globaux :

\begin{tabular}{|l|r|l|l|}
\hline & $\begin{array}{l}\text { Nombre de jugements } \\
\text { exclusifs de la } \\
\text { cohérence interne }\end{array}$ & $\begin{array}{l}\text { Nombre de jugement } \\
\text { de la cohérence interne } \\
\text { et du continuum }\end{array}$ & $\begin{array}{l}\text { Pas de } \\
\text { jugement }\end{array}$ \\
\hline en cours de lecture & 200 & $\mathbf{7 6}$ & $\mathbf{4 9}$ \\
\hline dans le commentaire final & $\mathbf{6 1 , 5 4 \%}$ & $\mathbf{2 3 , 3 8 \%}$ & $\mathbf{1 5 , 0 8 \%}$ \\
\hline & $\mathbf{1 1 2}$ & 205 & $\mathbf{8}$ \\
\hline
\end{tabular}

Modes de lecture sur l'ensemble des textes du corpus.

Ces résultats montrent deux faits saillants :

- en cours de lecture, plus de $61 \%$ des annotations s'intéressent à la suite produite par l'élève du point de vue de sa cohérence interne. Seuls $23 \%$ des évaluations font explicitement référence au texte-amorce au travers des remarques notées au fil du texte produit;

- en fin de lecture, ces tendances majeures s'inversent. Au moment de la rédaction de la remarque finale, $63 \%$ des appréciations comportent au moins une mention relative au texte-amorce. Cependant, plus de $34 \%$ des remarques ne concernent que le texte produit, même après la lecture complète du texte de l'élève.

Ces données, certes très générales, nous éclairent cependant sur la manière de lire une suite de texte; apparait une chronologie d'actions liée à des activations ou à des inhibitions d'informations relatives au texte-amorce. Il semble que les enseignants choisissent de manière différenciée les moments de la mise au premier plan d'une représentation sémantique ; dans ce cadre, nous avons relevé assez régulièrement un jugement évolutif du même texte par un évaluateur. Par exemple, un enseignant annote tout le texte de Précilia de façon négative (situation non mâ̂trisée; difficulté de maîtrise des temps, etc.) et conclut : suite réussie.

Ces modes différenciés de lecture posent selon nous la question du rapport entre niveau local et niveau global dans la construction de la cohérence en référence 
à l'idée de double composante horizontale et verticale dans l'interprétation : d'un côté, il s'agit de juger, au fur et à mesure de la mise en mémoire d'une unité sémantique, en quoi elle est liée à la précédente (processus ascendant); de l'autre, il s'agit d'activer des scénarios mais aussi des représentations de pratiques langagières et de les confronter au texte produit (processus descendant).

Nous avons choisi de présenter notre méthode d'investigation et de l'illustrer par un échantillonnage représentatif des remarques des enseignants. Nous avons souhaité montrer l'intérêt des lectures successives du corpus. En effet, cela nous a permis de constater 1) que les enseignants se sont approprié certains savoirs sur la question de la textualité 2) que l'objectivation des critères de cohérence ne réduit pas forcément le rapport singulier de chaque enseignant aux textes lus 3) que l'évaluation d'une suite de texte se réalise dans une tension entre une lecture incrémentale et une interprétation globale.

Nous allons conclure notre article en reprenant ces trois axes afin de les interroger en regard des définitions savantes et du point de vue de la formation.

\section{3. Écarts et accords entre les savoirs savants et les conceptions des enseignants : un problème pour la formation des maitres}

En ce qui concerne l'appropriation de certains savoirs sur la textualité, les définitions implicites :

- donnent une place réelle à la question de la textualité. Les enseignants mettent en œuvre leurs savoirs en linguistique textuelle pour évaluer les productions. Ils observent de façon tout à fait majeure les indicateurs linguistiques de référenciation et l'emploi des temps ;

- montrent que la construction de la cohérence relève du respect de contraintes interphrastiques plus que textuelles. L'évaluation des phénomènes de reprise est tout à fait significative, la recherche du référent s'opérant d'un point de vue strictement local et grammatical.

- posent le problème des liens constants entre le niveau syntaxique et le niveau textuel et montrent une lecture parfois trop strictement « pas à pas». Les remarques confirment que la phrase graphique est une unité d'intégration sémantique souvent traitée en soi et non en texte, en fonction d'un avant et d'un après textuel. La cohérence, au travers des annotations relevées, se construit lorsque les référents n'entrent pas en conflit, qu'ils se succèdent mais ne s'intercalent pas.

En ce qui concerne la dimension interprétative, les définitions implicites :

- accordent une place centrale au lecteur. Les annotations informent du fait qu'établir des liens de cohérence consiste à s'appuyer sur ses connaissances d'arrière-plan pour interpréter le contenu du texte lu;

- montrent conséquemment le rôle des inférences dans l'élaboration du sens, mais en valorisant les moins coûteuses. Les remarques des enseignants laissent apparaître qu'il existe deux types d'inférences : celles liées aux savoirs partagés et celles construites par liaison, c'est-à-dire par reconstruction d'éléments manquants mais inférables, à condition d'un certain coût cognitif. Pour la majorité des annotations exprimées, un texte cohérent est un texte pour lequel l'interprétation n'est pas coûteuse.

- mettent en avant le rôle des topics comme instruments métatextuels de construction de la cohérence, mais en montrant rarement la prise en compte de l'interprétation de l'élève. Les remarques montrent que dans le conti- 
nuum entre le texte-amorce et le texte construit, tous les enseignants ne répondent pas de façon univoque à la question du monde construit. " Ca ne parle pas de la même chose » pour chacun, ce qui provoque des sanctions ou des valorisations de mêmes objets du monde actualisés par le lexique choisi par l'élève. La recherche de l'intention de l'auteur n'apparaît que très rarement au travers des remarques.

En ce qui concerne le mode de lecture des enseignants, les annotations et remarques montrent

- une évaluation souvent en deux temps : d'abord une prise de connaissance du texte produit par l'élève, puis une évaluation de sa cohérence en regard du texte-amorce.

- que l'évaluation est traitée localement : lorsque le texte montre des problèmes locaux de lisibilité (syntaxe défaillante, mots permutés, problèmes de reprise de l'information), la tendance est à l'expression de remarques non pas essentiellement à propos des différentes contraintes de 1'exercice de continuité mais sur ces problèmes locaux. Cet état de fait conforte notre choix méthodologique initial de toilettage des textes afin d'inviter les enseignants à dépasser la difficulté locale majeure que représente l'orthographe et à annoter d'autres niveaux de la mise en texte.

- que l'évaluation semble toujours, pour certains enseignants, consister en la recherche des manques plus qu'en la valorisation des réussites. C'est la conséquence de ce regard porté sur les difficultés de « mise en grammaticalité » du texte, qui se lit par exemple en miroir dans l'évaluation en cours de lecture du texte de Samuel où nous comptons 39\% d'absence de jugement.

La position théorique implicite qui apparaît au travers des annotations des enseignants met en tension deux options définitoires de la cohérence : une option où le sens émane intrinsèquement des signes linguistiques et de leur agencement et une option où le sens se construit au fur et à mesure de l'interprétation successive des énoncés. Le premier cadre est retenu (1) en référence aux jugements des enseignants qui ont interprété les propositions des élèves en s'appuyant sur l'entour immédiat du segment interrogé (pour l'interprétation d'une anaphore par exemple) et/ou ont sanctionné une proposition lexico-sémantique sans montrer le souci de prise en compte du l'existence d'un lien entre le contenu du texte et le choix de l'expression de l'élève (2) en référence aux souhaits des enseignants d'obtenir une construction narrative homogène et constituée d'une structure stabilisée et complète. Le second cadre doit, nous semble-t-il, être retenu tout particulièrement du fait qu'il repose sur le principe de pertinence pour expliquer pourquoi un sujet va arrêter ou au contraire poursuivre son interprétation : le principe de pertinence envisage que l'interprétant réalise toujours le calcul le moins coûteux lui permettant de conclure qu'un discours est cohérent. Donc, d'énoncés en énoncés, il s'arrête à la première interprétation n'entrant pas en conflit avec les informations construites. Dans ce cadre, le lecteur juge la cohérence d'un texte à mesure de la facilité qu'il a à construire une intention globale au niveau du contenu à transmettre. Il apparaît que les remarques des enseignants engagent parfois l'activation du principe de pertinence et donc une présomption de cohérence dans le texte de l'élève, et d'autres fois au contraire, un « abandon » du fait d'un effort interprétatif trop coûteux.

La position des enseignants se réfère à des courants théoriques qui ont connu une évolution assez considérable en quelques décennies ; par exemple, avancer 
qu'ils s'appuient sur la « linguistique textuelle» pour juger la cohérence des textes doit être précisé : quand nous disons qu'ils convoquent la « linguistique textuelle », nous affirmons qu'ils se situent dans le cadre d'une recherche de bonne formation textuelle. Or, si la linguistique textuelle n'a pas modifié son objet central d'étude (ce que rappelle Adam 2005 : 29), elle l'a en revanche résolument inscrit dans une perspective plus large, prenant en compte les contextes de production et les formations socio-langagières. On peut donc conclure que si les enseignants s'appuient effectivement sur la linguistique textuelle, il s'agit plutôt de la conception didactisée depuis les années quatre-vingts sous l'appellation "grammaire textuelle » que de l'approche actuelle, plus complète et plus complexe, et dont la dénomination évolue entre « analyse textuelle des discours » et « pragmatique textuelle $»$.

La mise en perspective des définitions théoriques et des définitions en actes de la cohérence invite à nous poser certaines questions quant aux contenus didactisés en formation. Nous pensons que la formation pourrait peut-être prendre appui sur des savoirs disciplinaires élargis qui permettraient une analyse plus fine par les enseignants des enjeux de leur lecture-évaluation d'un texte d'élève : le jugement de cohérence, loin de se concentrer sur la liste des éléments morphosyntaxiques et lexicaux déclinés dans les programmes, s'appuie sur des configurations textuelles et sémantiques, des procédures psychologiques que les enseignants activent parfois de façon implicite. Un rôle de la formation au jugement de la cohérence de textes d'apprentis-scripteurs pourrait donc être l'accompagnement à la conscientisation de la manière dont chacun gère les différents niveaux de contrainte textuelle.

Comment entendons-nous « savoirs disciplinaires élargis»?

D'une part, la proposition d'exploration non seulement d'objets de savoir relatifs à l'enseignement du français, donc issus des sciences du langage et des théories littéraires, mais aussi de travaux en psychologie et psycholinguistique.

D'autre part, au sein de ces domaines de recherche, la proposition d'élargissement des théories d'appui, spécifiquement pour permettre :

- 1'analyse des enjeux textuels et/ou pragmatiques de faits de texte fondamentaux (par exemple la lecture d'une anaphore en fonction de son «avant» et de son « après »; la distinction entre la saillance locale et la saillance cognitive) ;

- la réflexion sur la tension entre variations et constantes génériques et lexico-sémantiques en jeu dans tout parcours interprétatif (par exemple les interactions entre le genre, les topics et l'isotopie ; le lien entre l'expérience et l'univers construit);

- la mise au jour des modes de lecture qu'implique l'évaluation de la suite d'un texte grâce à la connaissance de théories psychologiques (par exemple l'appel aux sources extérieures, le recours au texte en cours et l'empan de la mémoire de travail qui ont un rôle essentiel dans la construction de la cohérence).

Nous pensons que ces axes de recherche pourraient être problématisés dans le cadre de la formation des maitres. Seraient ainsi partagés et discutés des problèmes liés au fonctionnement et à la réception des textes dans le but d'une réflexion sur la manière d'évaluer un texte d'élève. Il s'agirait donc de l'apport d'un savoir pour enseigner et non pas à enseigner. 


\section{Références bibliographiques}

ADAM, J.-M. (1999) : Linguistique textuelle, des genres de discours aux textes. Paris : Nathan Université.

- (2002) : Cohérence. Dictionnaire d'analyse du discours. Paris : Seuil.

- (2005) : La linguistique textuelle, Introduction à l'analyse des discours. Paris : Armand Colin.

ApothéLOz, D. (1995) : Rôle et fonctionnement de l'anaphore dans la dynamique textuelle, Genève-Paris : Droz.

BRONCKART, J.-P. (1997): Activité langagière, textes et discours, Pour un interactionisme socio-discursif. Lausanne-Paris : Delachaux et Niestlé.

Charolles, M. (1995) : «Cohésion, cohérence et pertinence du discours », Travaux de linguistique, $n^{\circ} 29$, pp. 125-151.

— (2005) : «Cohérence, pertinence et intégration conceptuelle», Des discours aux textes modèles et analyses. P. Lane. (éd). Rouen : Publications des Universités de Rouen et du Havre, pp. 39-74.

- (2006) : « De la cohérence à la cohésion du discours », Cohérence et discours. F. Calas (dir). Paris : Presses de l'Université de Paris-Sorbonne, pp. 2538.

Chuy, M., Rondelli, F. (2010) : «Traitement des contraintes linguistiques et cognitives dans la construction de la cohérence textuelle », Langages. Paris : Armand Colin.

Combettes, B. (1995) : «Système de la langue et linguistique du texte », Le français dans tous ses états, $\mathrm{n}^{\circ} 30$.

Coirier, P., GaOnaC'H, D., PAsserault, J.-M. (1996) : Psycholinguistique textuelle, approche cognitive de la compréhension et de la production des textes. Paris : Armand Colin / Masson.

DUMORTIER, J.-L. (2005) : « Tout petit traité de narratologie buissonnière à l'usage des professeurs de français qui envisagent de former non de tous petits (et très mauvais) narratologues mais des amateurs éclairés de récits de fiction », Dyptique, $\mathrm{n}^{\circ} 3,116 \mathrm{p}$.

ECO, U. (1985) : Lector in Fabula. Le rôle du lecteur. Paris : Grasset.

ELALOUf, M.-L. (2005) : Ecrire entre 10 et 14 ans. Un corpus, des analyses, des repères pour la formation. Paris: SCEREN, $174 \mathrm{p}$.

FACQUES, B. (2002) : «Passé composé, imparfait et présent dans les récits journalistiques : des alternances aux ruptures temporelles », Cahiers Chronos, $\mathrm{n}^{\circ}$, pp. 105-133.

FAUCONNIER, G, TURNER, M. (2002): The way we think: conceptual blending and the maind's hidden complexities, Basic Books.

FRANÇOIS, F. (2004) : Enfants et récits, Mises en mots et «reste ». Textes choisis et présentés par Régine Delamotte-Legrand. France : Presses Universitaires du Septentrion, $226 \mathrm{p}$.

GÉRARD, F.-M.(2002) : «L'indispensable subjectivité de l'évaluation », Antipodes, $\mathrm{n}^{\circ} 156$, pp. 26-34.

GOMBERT, J.-E. (1997) : « Ontogenèse de la machine à lire », Cahiers de Beaumont, $n^{\circ} 74-75$, pp. $27-34$.

GRICE, H. P. (1979) : «Logique et conversation», Communications, $n^{\circ} 30$, pp. 57-72.

HALTÉ, J.-F. (1984) : «L'annotation des copies, variété ou base du dialogue pédagogique », Pratiques, n44, pp. 61-69.

Morillo Vincente, M. (2004) : «Sept contes de Michel Tournier», Revista Digital "Investigación y Educación », n8, pp. 2-14. 
Petitjean, A. (2007) : "Corpus et genres : quelles interactions ? », Discours, diachronie, stylistique du français, Etudes en hommage à Bernard Combettes. O. Bertrand, M. Charolles, J. François, C. Schnedecker (éds). Berne : Peter Lang, pp. 417-433.

PerRUCHet, P. (1989) Les automatismes cognitifs. Liège : Mardaga, 196 p.

PLANE, S. (2006) : « Singularités et constantes de la production d'écrit-L'écriture comme traitement de contraintes $»$ Diptyque, $n^{\circ} 5$, pp. 33-54.

RAstieR, F. (2001) : Arts et sciences du texte. Paris : Presses Universitaires de France.

— (2007) : «Du réalisme au postulat référentiel », Revue Texto! [en ligne] téléchargeable au format PDF : http://www.revue-texto.net/Inedits/Rastier/Rastier_Postulat.pdf

Rastier, F., Cavazza, M., ABeillé, A.(1994) : Sémantique pour l'analyse. Paris : Masson.

RONDELLI, F. (2008) : « La cohérence textuelle : rapport à la langue, à soi, à l'autre ? ", Actes des $\mathrm{X}^{\mathrm{e}}$ Rencontres Jeunes Chercheurs de l'école doctorale 268 Langages et Langues, 12 mai 2007. Paris : ILPGA.

— (2008) : «L'écriture des textes narratifs à l'école élémentaire : traitement des contraintes et jugements de cohérence », thèse non publiée, Université de Paris V-René Descartes, France.

- (2008) : «La cohérence des textes : entre préoccupations didactiques et questionnements linguistiques $», 1^{\text {er }}$ Congrès Mondial de Linguistique Française, publication en ligne : www.linguistiquefrancaise.org/10.1051/cmlf08139

SAUSSURE, Louis (de), STHIOUL, B. (2005) : «Imparfait et enrichissement pragmatique », Chronos, $\mathrm{n}^{\circ} 14$.

SPERBER, D. \&Wilson, D. (1989) : La pertinence. Paris : Editions de Minuit.

TURner, M. (2000), «L'imagination et le cerveau ; L'invention du sens », Conférences des 6 juin 2000 et 13 juin 2000 au Collège de France, Paris. Disponibles sur http://markturner.org/cdf/cdf1.html et http://markturner.org/cdf/cdf2.html

\section{Annexes}

\section{Annexe 1 : le texte amorce}

Le Kon-Tiki est un radeau qui fut construit pour un jeune savant norvégien. Ce radeau, semblable à ceux des ancêtres des Indiens du Pérou, lui permit, avec cinq compagnons, de traverser l'Océan Pacifique, sans moteur et sans voiles, en se laissant entraîner par les courants.

Un jour, Knut eut sans le vouloir une petite séance de natation avec un requin. Il nous était interdit de nous éloigner pendant notre bain à cause de la déviation du radeau, et à cause des requins. Mais un jour où la mer était particulièrement calme et où nous venions d'attraper les requins qui nous avaient suivis, une rapide trempette fut permise. Knut plongea le premier. Il s'était beaucoup éloigné avant de remonter à la surface pour revenir en nageant le crawl. A ce moment, nous vîmes une ombre plus grande que son corps monter derrière lui. Nous lançâmes un cri d'alarme, et il se dirigea de toutes ses forces vers le bord du radeau. Mais l'ombre appartenait à un nageur encore plus remarquable qui, s'élançant des profondeurs, gagnait sur Knut. Ils atteignirent l'embarcation en même temps. 


\section{Annexe 2 : les sept textes évalués}

\section{Texte de Précilia}

Ils atteignirent l'embarcation en même temps.

[é1] Joël, Jean-Laurent, Josselin, Jimmy essayèrent d'accélérer [é2] mais

le requin ne tombait pas. [é3] Jimmy prit une pelle [é4] et assomma le requin.

[é5] Josselin prit le téléphone [é6] et appela des spécialistes. [é7] Quelques heures plus tard

les spécialistes arrivèrent [é8] et ils leur posent quelques questions.

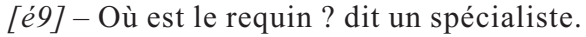

[él0]-Il est là ! crient les enfants en cœur.

[é11]-On s'en occupe! dirent les autres spécialistes.

[é12]-Pouvez-vous nous ramener? dirent-ils tous en cœur.

[é13]-Mais bien sûr!

[é14] Ils repartirent tous ensemble avec le requin [é15] et après quelques heures, ils entrèrent dans leur maison, contents de rentrer [é16] et ne voulurent

plus aller en mer!

\section{Texte de Samuel}

Ils atteignirent l'embarcation en même temps.

[él]-Attention, il te suit, cria Ethan.

[é2] Le requin essaya de le mordre [é3] mais en vain.

[é4] Knut sauta sur le radeau. [é5] Jarod prit un

bâton [é6] et tapait sur le requin. [é7] Mais celui-ci

cassa le bâton. [é8] A son tour, Darner prit un

autre bâton beaucoup plus long et plus dur

[é9] et le mit dans la bouche du requin [é10] quand celui

- ci l'ouvrit. [é11] Le requin, très gêné par le bâton

repartit dans les profondeurs de la mer.

[é12] - A présent, plus personne ne s'éloignera

du radeau, dit valmon.

\section{Texte de Valentine}

Ils atteignirent l'embarcation en même temps.

[él] C'était une sirène.

[é2] Une belle sirène avec une queue verte et un haut violet.

[é3] Et cette sirène avait des cheveux rouges.

[é4] Elle dit : «comment t'appelles-tu?

[é5]-je m'appelle Eric, [é6] et toi : [é7] je m'appelle Arielle.

[é8] Arielle dit : Tu veux aller voir mon père?

[é9] Eric dit: Mais je ne suis pas une sirène moi.

[é10] Arielle dit : je te donnerai une queue.

[é11] Et ils se marient.

\section{Texte de Camille}

\section{Ils atteignirent l'embarcation en même temps.}

[é1] Il lui lança la corde [é2] mais c'était trop tard à ce moment

là, Knut se fit manger par le requin, [é3] les autres

criaient « au secours, au secours » [é4] mais malheureu-

sement à ce moment là ils étaient en plein

milieu de l'océan.

[é5] - prenez les baïonnettes dit le capitaine

[é6] - Où tire-t-on capitaine

[é 7$]$ - en plein cœur «feu»

[é8] - nous avons tout tiré les munitions

[é9] - oh non! [é10] mais oui je pense il a celle de Knut

[é11] - Il n'y en a plus une munition capitaine

[é12] - Donnez la moi [é13] je ne la louperai pas!

[é14] 
[é15] A ce moment là le requin était

touché en plein cœur [é16] et ne remonta

jamais à la surface.

\section{Texte de Zoé}

Ils atteignirent l'embarcation en même temps.

[é1] Une fois arrivé [é2] le nageur saute dans le

radeau [é3] et les requins sautent avec lui. [é4] Knut dit :

[é5]-Arrête [é6] je n'en peux plus [é7] c'est toi qui a gagné

[é8] tu peux me tuer.

[é9] Le requin hésite et dit:

[é10]-je ne sais pas [é11] si je te tue ou pas [é12] tu es un homme

[é13] bon j'hésite.

[é14] Un peu plus tard le requin dit:

[é15]-j'ai pris ma décision [é16] mais je ne sais pas si j'ai raison

[é17] j'ai décidé que j'allais te tuer et tes hommes

en plus.

[é18] Fin

\section{Texte d'Alexandre}

Ils atteignirent l'embarcation en même temps. [él] L'un des

cinq compagnons prit une corde [é2] et l'attache sur le radeau.

[é3] La lance [é4] et Knut l'attrape. [é5] Les 4 autres prennent chacun une rame.

[é6] Il allait à la même vitesse que le requin.

[é 7$]$-Il dit merci les gars

[é8] Ils voient que la corde va bientôt lâcher. [é9] Le requin

n'a presque plus de force, [é10] il s'arrête 30 secondes.

[é11] Mais l'homme n'a pas le temps de remonter sur le radeau. [é12] Si il

monte sur le radeau [él3] le requin, va casser le ra-

deau [é14] et va manger ses copains. [é15] Alors il coupe la corde [é16] et

se sacrifie pour eux. [é17] Et après tout le monde n'avait

plus envie de naviguer. [é18] Ils ont vu une île. [é19] L'île de

Ko-Lanta [é20] et fabrique un autre radeau.

[é21]-Vraiment beau le radeau!!!

\section{Texte de Marvin}

Ils atteignirent l'embarcation en même temps.

[é1]-Knut remonte vite! [é2] Le requin est peut-être dans

les parages.

[é3] -Mais non, [é4] regarde la mer est toute calme !

[é5]-bon, fais comme tu veux !

[é6] Ils avancèrent dans le radeau.

[é7] Et tout un coup une tempête se leva. [é8]

- Oh le radeau va couler!!!

[é9] Et un requin remonta à la surface ?

[é10]-chut! [é11] Je crois qu'il y a un requin

[é12]-Et regarde Kant a disparu !

[é13] Je crois que c'est la fin.

[é14] - bon maintenant c'est à nous de jouer.

[é15] Et le lendemain, ils arrivèrent à l'autre

bout de l'océan. [é16] La ville était toute

calme.

[é17]- je ne me sens pas bien Kant?

[é18]-tant pis !

[é19] - Et Knut on a réussi notre épreuve.

[é20] Félicitation, Kant toi aussi Kneut

[é21] mais malheureusement Cyrus n'a pas

réussi. 
Annexe 3 : axes d'analyse des jugements de cohérence

\begin{tabular}{|c|c|c|}
\hline \multirow{4}{*}{ 氡 } & $\begin{array}{l}\text { Jugements de la dimension } \\
\text { socio-pragmatique }\end{array}$ & $\begin{array}{l}\text { - Rapport à la consigne } \\
\text { - Prise en compte du texte-amorce } \\
\text { - Identification de l'acte de parole global }\end{array}$ \\
\hline & $\begin{array}{l}\text { Jugements de la dimension } \\
\text { énonciative }\end{array}$ & $\begin{array}{l}\text { - Type d'ancrage énonciatif } \\
\text { - Cohérence énonciative } \\
\text { - voix } \\
\text { - polyphonie } \\
\text { - micro - actes de discours }\end{array}$ \\
\hline & $\begin{array}{l}\text { Jugements de la dimension } \\
\text { structurelle }\end{array}$ & $\begin{array}{l}\text { - Plan du texte } \\
\text { - Séquences / périodes } \\
\text { - Procédés textuels } \\
\text { - Texture micro - linguistique } \\
\end{array}$ \\
\hline & $\begin{array}{l}\text { Jugements de la dimension } \\
\text { thématique }\end{array}$ & $\begin{array}{l}\text { - Domaines sémantiques } \\
\text { - Thèmes et sous - thèmes } \\
\text { - Réseaux lexicaux } \\
\text { - Raconté réaliste / imaginaire }\end{array}$ \\
\hline \multirow{2}{*}{ 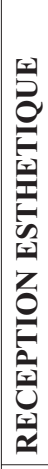 } & $\begin{array}{l}\text { Rapport au contenu } \\
\text { et à l'expression }\end{array}$ & $\begin{array}{l}\text { Appréciation : } \\
\text { - des reprises - modifications liées à la culture de l'élève } \\
\text { - de la signification trouvée qui relève ou non du canonique } \\
\text { - des techniques d'intéressement } \\
\text { - de la disposition intentionnelle }\end{array}$ \\
\hline & & $\begin{array}{l}\text { Appréciation des faits : } \\
\text { - de brièveté } \\
\text { - d'ellipses } \\
\text { - d'onomatopées } \\
\text { - de parataxe } \\
\text { - de sonorités }\end{array}$ \\
\hline \multicolumn{2}{|r|}{$\begin{array}{l}\text { Jugement de cohérence et parcours } \\
\text { de lecture }\end{array}$} & $\begin{array}{l}\text { Jugement en cours de lecture et } \\
\text { Jugement après lecture }\end{array}$ \\
\hline
\end{tabular}

